\title{
PLASMA MOTIONS AROUND SUNSPOTS
}

\author{
G. LUSTIG ${ }^{1}$ AND H. WÖHL ${ }^{2}$ \\ ${ }^{1}$ Institut für Astronomie, Karl-Franzens-Universität \\ A-8010 Graz, Austria \\ ${ }^{2}$ Kiepenheuer-Institut für Sonnenphysik \\ D.W7800 Freiburg, Germany
}

\begin{abstract}
Motions of large, stable sunspots - which are in some cases even recurrent - are determined using the Vacuum Tower Telescope (VTT) at Tenerife and sunspot drawings taken at the Kanzelhöhe Observatory in Austria. Around the sunspots fields of about 100 by 100 arcseconds are covered by a scanning spectrograph entrance slit in steps of one arcsecond every 15 seconds to determine the plasma velocities. The main aim is a determination of plasma and sunspot velocities within larger fields of activity and a search for special patterns of plasma flows, which reflect the well-known braking of the sunspot rotation velocity during aging.
\end{abstract}

\section{SCIENTIFIC BACKGROUND}

Ten years ago Balthasar et al.(1982) demonstrated the braking of the solar rotation velocity of stable, recurrent sunspots from the Greenwich data. A braking of the order of $0.3[\mathrm{~m} / \mathrm{s}]$ to $0.8[\mathrm{~m} / \mathrm{s}]$ per day was determined. That publication contained also some theoretical investigations concerning the braking mechanisms.

In the meantime several publications appeared reporting the braking of the solar rotation for all types of sunspots with the aging of the spots. A typical example for these new results is the publication by Zappala and Zucarello (1991).

Already 15 years ago Beckers (1977) claimed, that the solar rotation velocity of the plasma inside a sunspot is of the same order as the solar rotation velocity of the sunspot from tracer measurements. His sample of spots was rather small - just 5 spots.

Koch (1984) showed that the solar rotation velocity of plasma within sunspots was $30[\mathrm{~m} / \mathrm{s}]$ to $60[\mathrm{~m} / \mathrm{s}]$ more rapid than the plasma around the sunspots. He also verified, that the rotation velocity of the sunspots determined by the tracer technique were of the same order as the plasma velocities determined by the Doppler effect within the sunspot umbrae. The measurements of Koch were performed using a pinhole in the focal plane of the telescope, therefore only at a few positions east and west of the sunspots 
velocity data were taken - at that time by scanning of the spectra with a multiplier behind a moving slit. His sample was also just 5 sunspots.

\section{SCIENTIEIC AIMS}

The general aim of the project is to determine influences of the braking of sunspots on the plasma flow around them. The mean flow pattern around stable - and hopefully recurrent (see above) - sunspots shall be determined.

Important parameters are the rotation velocities of the sunspots with respect to the surrounding plasma and its velocity. In addition e.g. the magnetic field distribution in that area is of importance.

Expected variations of the velocity field patterns are of the order of some ten meters per second, therefore high precision spectral line position measurements with reference lines are needed.

\section{OBSERVING PRQCEDURES}

The main measurements were performed using the Vacuum Tower Telescope (VTT) and its Echelle grating spectrograph of the Observatorio del Teide at Izaña/Tenerife/Spain. The Doppler shifts of the central core of the non split Fe $5576.1 \AA$ line were determined with respect to 8 iodine lines from a laboratory absorption cell. The position of the cell was in front of the southern part of the spectrograph's entrance slit. The solar and laboratory spectra were recorded simultaneously on large CCDs with $1024 * 1024$ pixels each.

Around the sunspots fields of about $100 * 100$ arcseconds were covered by a scanning spectrograph entrance slit in steps of one arcsecond every 15 seconds to determine the plasma velocities. The scanning time was in general one hour. Stable spots between about 40 degrees and 80 degrees distance to the central meridian were used in the center of the scanned velocity field areas. Depending on the weather conditions and other observing projects for high spatial resolution up to 9 complete area scans could be performed during one observing day. A maximum of 11 areas around one spot could be scanned such sunspots were in general detected first at the east limb, but it was aimed to observe around a similar number of spots from the eastern and the western hemispheres of the Sun.

In summer 1992 two large CCD cameras were available, therefore it was decided to collect simultaneously the spectral region at $6301 \AA$ to $6303 \AA$, which includes two splitting solar lines and two terrestrial molecular oxygen lines for reference. There were several reasons to do so:

The splitting lines allow to give also the magnetic field strength distribution - at least in the spots and in active areas.

Since several solar plasma velocity measurements - e.g. recently the solar polar differential rotation measurements by Wöhl and Ye (1990) were performed using these lines, it is of interest to compare simultaneous measurements taken with the non split and the split lines and the different reference systems. 


\section{SUNSPOT POSITION MEASUREMENTS}

The VTT was used for the determination of the sunspot positions many times a day - at least before and after each area scan. But for the whole project other information is more important:

During preparatory measurements in 1990 the guiding quality of the three main solar telescopes at Izaña - the Gregory Coudé Telescope, the Vacuum Newton Telescope and the VTT - as well as the possibility to use them for absolute sunspot position determinations was compared with respect to dedicated instruments in Austria and Hungary (Alvensleben et al., (1990)).

To determine the tracer velocities of the sunspots for this project it was decided to use mainly the data from the Kanzelhöhe observatory in Austria in addition to the position data obtained at the VTT. The main reason was the availability of more than one observation per day during the passages when measurements at the VTT were performed. In addition the access of the data before and/or after our Doppler measurements - for a better determination of the braking - is easy.

Some data from german amateur groups are used for comparison.

\section{OBSERVATIONS AND REDUCTIONS PERFORMED}

In summer 1990, when the quality of the telescopes was determined, also the first few plasma measurements were performed.

The next observing periods were in June and August 1991, when most of the plasma velocities, which are now available, were recorded. A total of about 70 fields was scanned.

The last observing period was this year, from the middle of June until begin of July 1992, and a total of about 60 areas were scanned.

The data reduction and all the necessary corrections for relative motions of the Earth and the Sun, the limb shift of the spectral line and the geometrical conversions necessary were performed for each point within the velocity field using the SUN RISC workstation network of the Kiepenheuer Institute. The codes were programmed in IDL (Interactive Data Language). No details will be given here, but just the remark, that flat field images were constructed from the scans through quiet areas at the beginning or end of each scan. About 40 images were combined for a flat field.

The minima, maxima and averages from the velocity maps as well as standard deviations were computed. These data were compared with results for the plasma and the sunspot solar rotation measurements published in the literature.

In addition artificial images were constructed by sampling the continuum intensities from all the spectra and putting them in arrays of the same format as the velocity field data. Examples will be given below.

The sunspot position data were used to determine solar rotation velocities for the sunspots.

These general reduction procedures are performed for all Doppler measurements available as well as for all sunspot position data from 1990 and 1991. The position data from 1992 are only reduced for the VTT up to now. 


\section{DETAILED ANALYSES AND PRESENT RESULTS}

The data from the complete observing period for one sunspot from August 1991 were reduced in detail: The intensity and velocity maps were plotted using surface, contour and false image techniques. Averages of selected areas were determined and a higher rotation velocity of the plasma on the western side of the sunspot was found as compared to the eastern side. This spot (Mt. Wilson group No. 27019) was scanned eight times August 23 until 26, 1991. There are two days with two observations each (23rd and 25th), one with three observations (24 th) and only one with a single scan (26th). From the Solar Geophysical Data a classification as $\mathrm{H}$ type spot was given for most of the time during that passage.

The sidereal rotation velocity determined from $34 \mathrm{VTT}$ position observations for the same period is $(14.32 \pm .58)$ [deg/day], while it is $(14.24$ \pm .16) [deg/day] from the Kanzelhöhe position observations. For the latitude of -9 degrees this equals to a rotation velocity of $1987[\mathrm{~m} / \mathrm{s}]$ and $1976[\mathrm{~m} / \mathrm{s}]$, respectively.

The plasma velocity within the spot is $(1905 \pm 30)[\mathrm{m} / \mathrm{s}]$ from seven of the eight scans. The plasma velocity is $(1998 \pm 19)[\mathrm{m} / \mathrm{s}]$ in the penumbra (this is not corrected for an influence of the Evershed effect) and it is (1843 $\pm 20)[\mathrm{m} / \mathrm{s}]$ within an area with a maximum distance of 1.8 degrees from the umbral minimum intensity center.

The data from a single area scan of the observations from June 1992 were reduced to compare the velocity fields determined with the three solar lines. Three plots through these fields show the high correlation of the velocities outside the sunspots. Again there is a clear indication for a higher rotation velocity of the plasma on the west side as compared to the east side of the sunspot.

\section{FURTHER REDUCTIONS AND OBSERVING PROJECTS}

More sophisticated methods will be used to extract the mean flow pattern or its characteristics depending on selected parameters. In addition the magnetic fields will be determined. The possible influences of changing observing conditions (e.g. scattered light) and solar atmospheric parameters (e.g. the changes of the limb effect within active regions) will be evaluated. Attempts to determine the flow pattern from granular motions will be continued in the near future in parallel to a few further measurements using very stable spots.

\section{REFERENCES}

Alvensleben, A. von, Casas, R., Csepura, G., Lustig, G., Otruba, W., Schroll, A., Vazquez, M., Wittmann, A.D., Wöhl, H.: 1990, Astron.Ges. Abstract Series 5,43

Balthasar, H., Schüssler, M., Wöhl, H.: 1982, Solar Phys. 76, 21

Beckers, J.M. : 1977, Astrophys. J. 213, 900

Koch, A.: 1984, Solar Phys. 93, 53

Wöhl, H., Ye, B.: 1990, Astron. Astrophys. 240, 511

Zappala, R.A., Zucarello, F. : 1991, Astron. Astrophys. 242, 480 